This is the accepted version of the following article:

Rabti A., Mayorga-Martinez C.C., Baptista-Pires L., Raouafi N., Merkoçi A.. Ferrocene-functionalized graphene electrode for biosensing applications. Analytica Chimica Acta, (2016). 926. : 28 - . 10.1016/j.aca.2016.04.010,

which has been published in final form at https://dx.doi.org/10.1016/j.aca.2016.04.010 () https://dx.doi.org/10.1016/j.aca.2016.04.010. This manuscript version is made available under the CC-BY-NC-ND 4.0 license http://creativecommons.org/licenses/by-nc-nd/4.0/ 


\section{Ferrocene-functionalized graphene electrode for biosensing applications}

Amal Rabti, ${ }^{a, b, \ddagger}$ Carmen C. Mayorga-Martinez, ${ }^{a}$ Luis Baptista-Pires, ${ }^{a}$ Noureddine Raouafi ${ }^{*}$ and Arben Merkoçi ${ }^{* a, c}$

a. Catalan Institute of Nanoscience and Nanotechnology (ICN2), CSIC and The Barcelona Institute of Science and Technology, Campus UAB, Bellaterra, 08193 Barcelona, Spain.

b. Université de Tunis El-Manar, Faculté des Sciences de Tunis, Laboratoire de Chimie Analytique et Electrochimie (LR99ES15), Campus universitaire de Tunis ElManar, 2092, Tunis, Tunisia.

c. ICREA, Barcelona, Catalonia, Spain.

Corresponding authors:

Email: arben.merkoci@icn.cat (Arben Merkoçi), Fax: +34935868020; Tel: $+34935868014$

Email: n.raouafi@fst.rnu.tn (Noureddine Raouafi), Fax: +21671883424; Tel: +21655985599.

\section{Abstract}

A novel ferrocene-functionalized reduced graphene oxide ( $\mathrm{rGO}$ )-based electrode is proposed. It was fabricated by the drop casting of ferrocene-functionalized graphene onto polyester substrate as the working electrode integrated within screen-printed reference and counter electrodes. The ferrocene-functionalized $r G O$ has been fully characterized using FTIR, XPS, contact angle measurements, SEM and TEM microscopy, and cyclic voltammetry. The XPS and EDX analysis showed the presence of $\mathrm{Fe}$ element related to the introduced ferrocene groups which is confirmed by a clear CV signal at ca. $0.25 \mathrm{~V}$ vs. AgCl/Ag. Mediated redox catalysis of $\mathrm{H}_{2} \mathrm{O}_{2}$ and bio-functionalization with glucose oxidase for glucose detection were achieved by the bioelectrode providing a proof for potential biosensing applications.

\section{Keywords}

Reduced graphene oxide electrode; Functionalization; Ferrocene; Non-enzymatic Sensor; Biosensor. 


\section{Introduction}

Graphene derivatives are widely used as modifiers of conventional electrodes such as glassy carbon [1-2] and screen-printed carbon [3-4] electrodes to prepare electrochemical (bio)sensing devices. Drop casting [5], adsorption [6] and electrodeposition [1] are the most popular modification methods. However, modifying such surfaces with graphene derivatives undergoes a complex process and would potentially add summative effects to the underlying electrodes [7]. The observed electrochemical behavior is indeed hard to understand since graphene own contribution is not clear due to exposed areas of underlying electrodes.

Alternatively, using graphene as a "standalone" electrode material is highly regarded to fully exploit the graphene high conductivity. The "freestanding" graphene films are accessible via numerous approaches including spin coating, vacuum filtration, pressing graphene aerogel or hydrogel, chemical vapor deposition and screen and inkjet printing [8-15]. Since the electrode performances are intimately related of the ink composition, searching for inks with suitable properties remains a challenging research task. Until now, using a graphene oxide (GO)-based ink is still the dominant strategy for the preparation of graphene films thanks to its good dispersibility in a large variety of solvents. However, some of these solvents are toxic and some others need long time to dry while aqueous dispersion requires surfactants that may need a further removal step because of their insulating character [15]. Given the insulating nature of GO [16] reduction using hydrazine [8] thermal annealing [14] electrochemical reduction [3] or laser irradiation [5] is primordial to regenerate the graphene conductivity.

So far, many applications of graphene in physics have been devised [17] but applications in chemistry, biochemistry and biosensing still need development. Despite the huge number of reported electrochemical sensors based on graphene materials [18], screen-printed graphene electrodes (SPGE) have been only used for the detection of small biologically relevant analytes. In fact, ascorbic acid (AA), dopamine (DA) and uric acid (UA) can be sensitively and selectively detected by SPGE [19]. Moreover, these electrodes were used for the sensing of $\beta$-nicotinamide adenine dinucleotide, AA, UA and DA [13]. Still, electrochemical biosensors are difficult to achieve without the adequate electronic mediator. 
Ferrocene is the most used organometallic compound in the design of electrochemical sensors thanks to its good electrochemical behavior [20]. However, there are a limited number of reports on ferrocene-modified graphene electrodes. Although drop casting or electrodeposition of ferrocene-functionalized graphene using covalent or non-covalent methods onto glassy carbon electrodes is interesting [21-24], these methods inherently suffer from several problems such as the lack of fabrication control. Graphene/ferrocene composite carbon paste electrode [25] and 1(4-bromobenzyl)-4-ferrocenyltriazole/ionic liquid-modified graphene paste electrode with renewable surfaces have been prepared [26]. However, to the best of our knowledge, no ferrocene-functionalized graphene ink for the preparation of graphene "standalone" electrode has been reported.

Thus, this work proposes a new functionalized rGO electrode for (bio)sensing application. The $[1,2]$-cycloaddition of newly prepared $\omega$-azidodiethyleneglycol- $\alpha$ ferrocenylcarboxylic acid ester (agFc) and reduced graphene oxide nanosheets yielded highly water dispersible agFc-rGO nanosheets as schematized in Figure 1a. The agFc-rGO mixed with chitosan (CS) forms an excellent electroactive carbon ink for the electrode preparation (Fig. 1b). The new material has been fully characterized using spectroscopic techniques, electron microscopy, contact angle measurements and cyclic voltammetry. The electrode was used to mediate redox reaction of $\mathrm{H}_{2} \mathrm{O}_{2}$ and when it is biofunctionalized with glucose oxidase it can serve to detect glucose. 

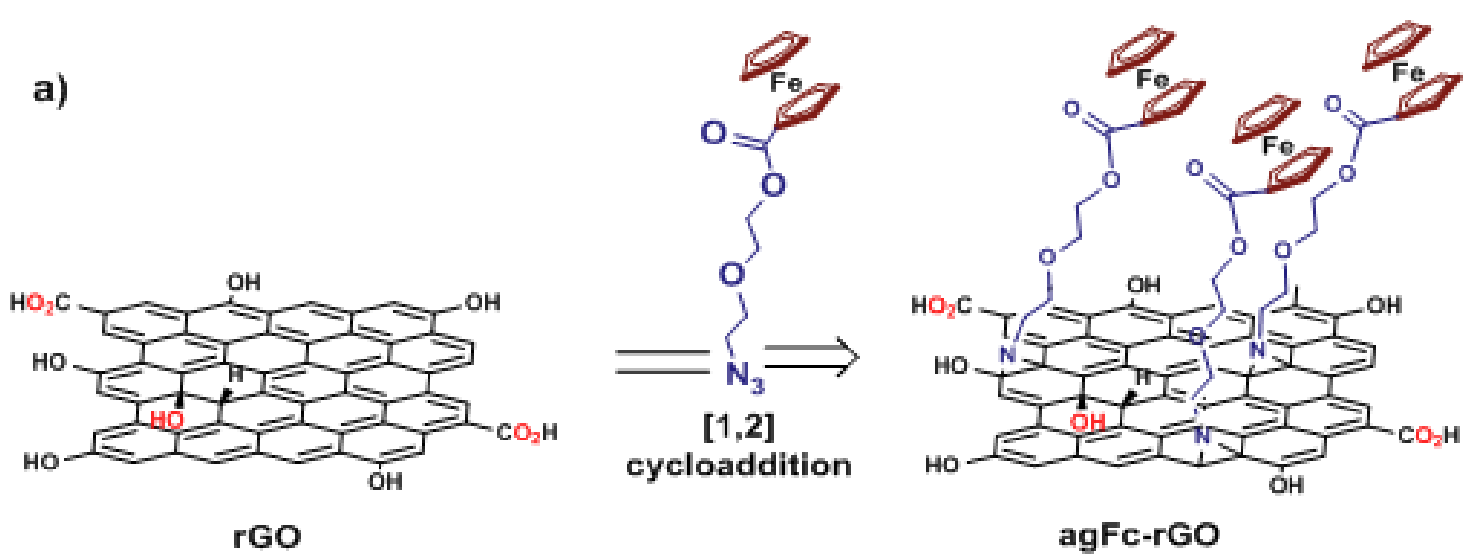

b)
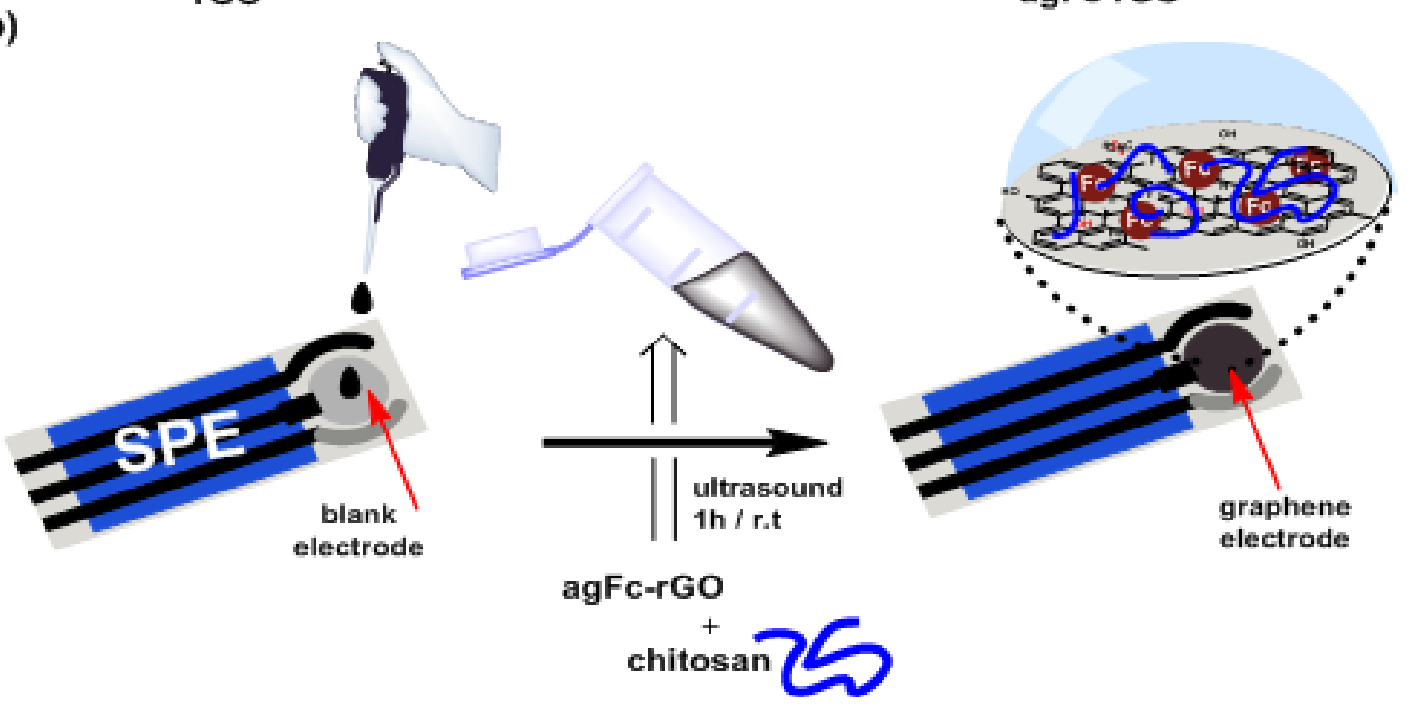

Fig. 1. a) Schematic method for the preparation of agFc-rGO and b) Preparation of agFc-rGO electrode by drop-casting of agFc-rGO/chitosan composite onto the PET substrate pre-integrated in electrochemical cell formed by screen-printed reference and counter electrodes.

\section{Experimental}

\subsection{Materials and Methods}

Diethylene glycol ( $>98 \%)$, tosyl chloride ( $>99.5 \%)$, sodium hydroxide ( $>99 \%), \mathrm{HCl}(37$ wt.\%), sodium azide (98\%), ferrocenylcarboxylic acid (97\%), dimethylaminopyridine (DMAP) (99\%), dicyclohexylcarbimide (DCC) (99\%), phosphate-buffered saline (PBS) tablets, chitosan, hydrogen peroxide (30\%), glucose oxidase (from Aspergillus niger), glucose, silica gel 60 mesh, aluminium TLC plates and all used solvents were of analytical grade purchased from Sigma-Aldrich and used as received. Graphene oxide (5 mg.mL ${ }^{-1}$ ) solution was purchased from Angstron Materials (OH, USA). 
Deionized water (>18.2 M $2 . \mathrm{cm}$ ) used for all the aqueous preparations was produced by means of a Milli-Q, Millipore system.

Cyclic voltammetry (CV), differential pulse voltammetry (DPV) and chronoamperometry (CA) were recorded using a PC-controlled Metrohm Autolab PGSTAT $302 \mathrm{~N}$ electrochemical workstation in 0.1M PBS solution as supporting electrolyte. Electrochemical impedance spectroscopy (EIS) measurements were performed in a $0.1 \mathrm{M} \mathrm{KCl}$ solution containing $5.0 \mathrm{mM}\left[\mathrm{Fe}(\mathrm{CN})_{6}\right]^{4 / 3-}$ with a frequency ranging from $100 \mathrm{kHz}$ to $0.1 \mathrm{~Hz}$ using an Autolab M204 equipped with FRA32 module. Experiments were designed and data collected using Nova $\AA^{\circledR}$ software.

All inks used for the screen-printing of electrodes were purchased from Acheson Industries (Germany). A sequential method was used to deposit a graphite-based ink, an $\mathrm{Ag} / \mathrm{AgCl}$ ink then a insulating ink on a polyester substrate (Autostat HT5 polyester sheet, McDermid Autotype, U.K). After the deposition of each layer, a drying process followed by keeping the polyester substrate at $120^{\circ} \mathrm{C}$ for either $45 \mathrm{~min}$ (graphite) or $30 \min (\mathrm{Ag} / \mathrm{AgCl}$ and insulator). Moreover, two kinds of masks were used: one having a blank working electrode to be used for agFc-rGO electrode preparation and the other containing a working electrode to print SPCE with a $3 \mathrm{~mm}$ diameter.

SEM and (S)TEM micrographs were recorded using a FEI Quanta 650 FEG Environmental scanning electron microscope and high-resolution FEI Magellan 400L XHR, respectively. Energy-dispersive X-ray spectroscopy (EDX) was recorded using Oxford Instruments Xmax $20 \mathrm{~mm}^{2}$. Mid-infrared spectra were acquired with a Varian 670-IR spectrometer equipped with a DTGS (deuterated tryglicine sulfate) detector. The spectral resolution used for all experiments was $4 \mathrm{~cm}^{-1}$ using attenuated total reflection (ATR) mode. Infrared spectra were recorded in the range of 4000-600 $\mathrm{cm}^{-1}$.

X-ray photoelectron spectroscopy (XPS) measurements were performed with a Phoibos 150 analyzer (SPECS $\mathrm{GmbH}$, Berlin, Germany) in ultra-high vacuum conditions with a monochromatic aluminum Ka x-ray source $(1486.74 \mathrm{eV})$. The energy resolution as measured by the FWHM of the Ag $3 d 5 / 2$ peak for a sputtered silver foil was $0.58 \mathrm{eV}$. X-ray diffraction (XRD) measurements were performed on a Siemens D-5000 X-ray diffractometer with graphite monochromatized $\mathrm{Cu}-\mathrm{Ka}$ radiation. Patterns were recorded over the $2 \theta$ range of $10-80^{\circ}$ at $40 \mathrm{kV}$ and $40 \mathrm{~mA}$. 
Easy drop contact angle measuring instrument was used to perform contact angle of the prepared electrodes.

\subsection{Preparation of reduced graphene oxide ( $\mathrm{rGO}$ ) and ferrocene-functionalized reduced graphene oxide (agFc-rGO) nanosheets}

To reduce graphene oxide, $2 \mathrm{~mL}$ of commercial GO were mixed with $8 \mathrm{~mL}$ of deionized water to obtain a $1 \mathrm{mg} \cdot \mathrm{mL}^{-1}$ solution. Then, $0.5 \mathrm{~g}$ of $\mathrm{NaOH}$ was added and the mixture was refluxed for $1 \mathrm{~h}$. After cooling to room temperature (RT) and centrifugation of the mixture for $30 \mathrm{~min}$, the supernatant was removed, $10 \mathrm{~mL}$ of ultrapure water and $1.25 \mathrm{~mL}$ of $\mathrm{cc}$. $\mathrm{HCl}$ were added and the mixture was refluxed again for $1 \mathrm{~h}$. After cooling to RT, the mixture was separated by repeated centrifugation and washed with water and acetone, affording $\mathrm{rGO}$ which was further dried in the oven overnight at $60^{\circ} \mathrm{C}$.

rGO (5 mg) and N-methylpyrrolidine (NMP) (5 mL) were placed in a $10 \mathrm{~mL}$ Schlenk flask fitted with a condenser. The mixture was treated with an ultrasonic bath for $1 \mathrm{~h}$. After the mixture was bubbled with nitrogen for $15 \mathrm{~min}, 150 \mathrm{mg}$ of agFc were added. The mixture was then heated at $160{ }^{\circ} \mathrm{C}$ for $24 \mathrm{~h}$ under $\mathrm{N}_{2}$ atmosphere. The solution was allowed to cool to RT then the modified graphene was separated by repeated centrifugation and washed with acetone.

\subsection{Preparation of agFc-rGO electrode}

An amount of $0.2 \mathrm{mg}$ of agFc-rGO was introduced into $50 \mu \mathrm{L}$ of a CS solution and the mixture was sonicated for $1 \mathrm{~h}$. Then, $5 \mu \mathrm{L}$ of the resulting solution were spread evenly onto the PET in the working electrode area and allowed to dry at RT. This protocol is used in the entire work unless otherwise indicated. For the fabrication of glucose biosensors, $10 \mu \mathrm{L}$ of the enzyme GOx (20 mg. $\left.\mathrm{mL}^{-1}\right)$ dissolved in PBS were casted onto agFc-rGO layer and was dried at RT. A third layer of a $5 \mu \mathrm{L}-\mathrm{CS}$ solution was added and let to dry. The biosensors were stored at $4{ }^{\circ} \mathrm{C}$ when not in use.

\subsection{Electrochemical Measurements}

All electrochemical measurements were carried out in triplicate by immersing the modified agFc-rGO electrode in $2 \mathrm{~mL}$ of $0.1 \mathrm{M} \mathrm{N}_{2}$-saturated PBS $(0.1 \mathrm{M}, \mathrm{pH}=7)$ as the supporting electrolyte. For all the experiments, the CV curves were measured at 
a scan rate of $100 \mathrm{mV} . \mathrm{s}^{-1}$ for potential range comprised between -0.1 and $0.7 \mathrm{~V}$. For chronoamperometry, the electrocatalytic detection of $\mathrm{H}_{2} \mathrm{O}_{2}$ was obtained by applying a potential of $+0.6 \mathrm{~V}$ for $250 \mathrm{~s}$. The DPV was performed by scanning in the potential range comprised between 0.0 and $0.4 \mathrm{~V}$ (step potential $10 \mathrm{mV}$, modulation amplitude $25 \mathrm{mV}$, scan rate $100 \mathrm{mV} \cdot \mathrm{s}^{-1}$ ). After each addition of $\mathrm{H}_{2} \mathrm{O}_{2}$ or glucose, the cell solution was stirred for $60 \mathrm{~s}$. All electrochemical experiments were carried out at room temperature.

\section{Results and discussion}

\subsection{Preparation and characterization of reduced graphene oxide}

The reduction process for the commercial GO sheets was performed in a 2-step mild thermal annealation using $\mathrm{NaOH}$ and $\mathrm{HCl}$ to avoid the incorporation of nitrogen/boron or additional alcohols into the graphene structure as when hydrazine or sodium borohydride are used [27-28]. Removal of the abundant hydroxyl and epoxide functionalities was confirmed by FTIR analysis (Fig. 2). In fact, IR spectrum (a) related to GO shows the presence of various oxygen functional groups i.e. vo-H at $3500-3000 \mathrm{~cm}^{-1}, v \mathrm{C}=\mathrm{O}$ at $\mathrm{ca} .1730 \mathrm{~cm}^{-1}$ and $v \mathrm{c}-\mathrm{o}$ at $1000-1200 \mathrm{~cm}^{-1}$ and sp'-hybridized $\mathrm{C}=\mathrm{C}$ vibrations. In contrast, the IR spectrum (b) of $\mathrm{rGO}$ shows that the intensity of the hydroxyl large band at $\sim 3000-3500 \mathrm{~cm}^{-1}$ and the C-O band at $\sim 1050-1150 \mathrm{~cm}^{-1}$ are much lower. The persistence of the carbonyl band at $\sim 1750 \mathrm{~cm}^{-1}$ originated from ketones or quinones as these are stable in basic conditions.

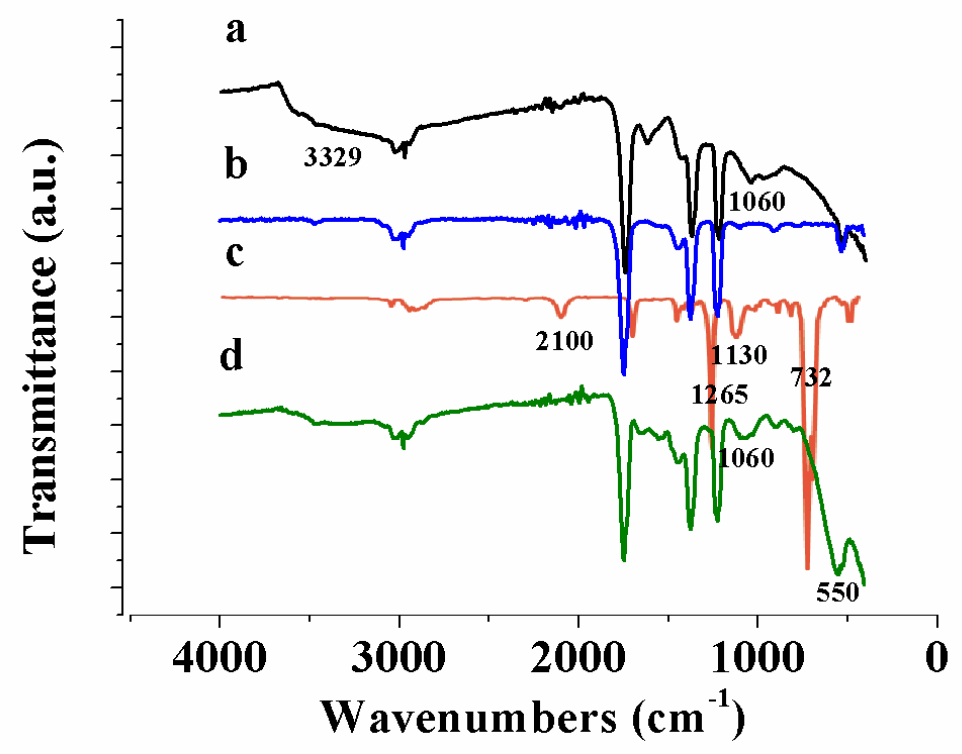


Fig. 2. FTIR spectra of $\mathrm{GO}(\mathrm{a}), \mathrm{rGO}(\mathrm{b}), \operatorname{agFc}(\mathrm{c})$ and agFc-rGO (d)

To further explore the composition of the materials, we resorted to XPS (Fig. 3a-b). The deconvoluted C1s XPS spectrum shows two large peaks at 284.8 and $286.7 \mathrm{eV}$ corresponding to the contributions from $\mathrm{C}=\mathrm{C}$ and $\mathrm{C}-\mathrm{O}$ of hydroxyl and epoxide functionalities, respectively. Two other small shoulders, appearing at 287.4 and 288.5 $\mathrm{eV}$, are attributed to $\mathrm{C}=\mathrm{O}$ double bond components of carboxyl and ketone functionalities. The chemical removal of oxygenated functionalities from the GO was confirmed by the decrease of the Ic-o/lc-c ratio which varies from 1.12 to 0.51 .

TEM of rGO showed evidences of typical multilayer graphene sheets with wrinkled structures (Fig. 3c-d). The contact angle measurements of GO and rGO were evaluated to be $20.9^{\circ}$ and $98.3^{\circ}$, respectively (Fig. S1, ESI†). Apparently, such a hydrophobic surface of rGO was the result of the obvious loss of oxygenated groups in $\mathrm{GO}$ after reduction. Furthermore, X-Ray diffraction patterns support the reduction of $\mathrm{GO}$ by the shift of the diffraction peak from $2 \theta=18.7^{\circ}(\mathrm{d}=0.47 \mathrm{~nm})$ to $2 \theta=28.5^{\circ}$ $(d=0.31 \mathrm{~nm})$, suggesting the reestablishment of the conjugated graphene network (Fig. S2). 
a)

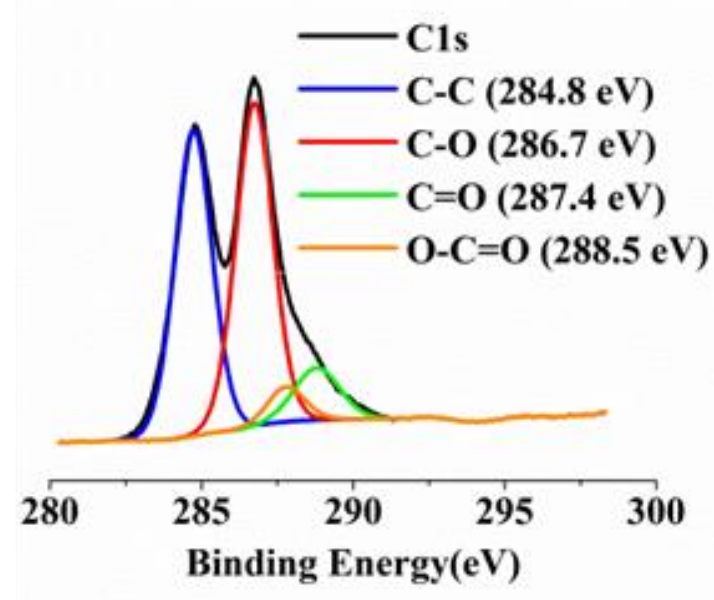

\section{c)}

$1 \mu \mathrm{m}$ b)

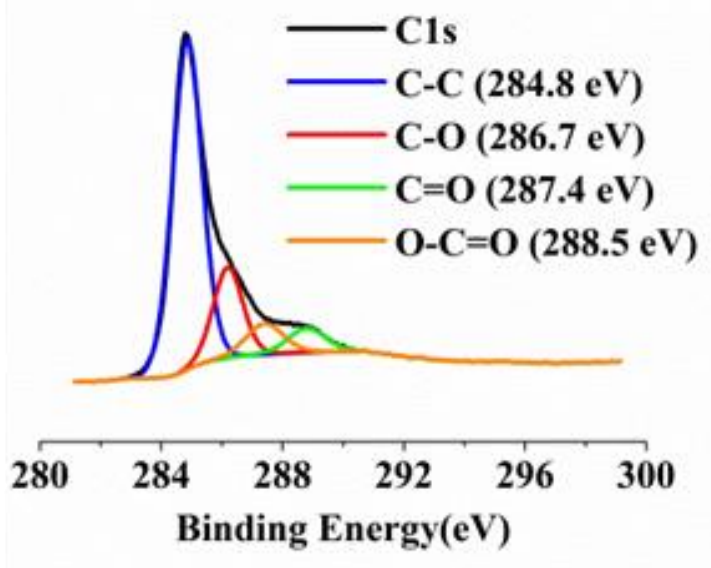

d)

$1 \mu \mathrm{m}$

Fig. 3. The de-convoluted XPS spectrum of the C 1s peaks for GO (a) and rGO (b); (S)TEM images of the GO (c) and rGO (d).

\subsection{Preparation of the ferrocene-functionalized rGO}

\subsubsection{Preparation of agFc-rGO}

The agFc compound was synthetized in few straightforward steps using a procedure adapted from literature [29]. To prepare the mono-azidodiethyleneglycol, a DMAPcatalyzed Steglich esterification was used to introduce the ferrocene group (Scheme $\mathrm{S} 1$, ESI†). Refluxing $\mathrm{rGO}$ and agFc in $\mathrm{N}$-methylpyrrolidine NMP for 24 hours yielded highly water-dispersible agFc-rGO nanosheets as illustrated in Figure 1a via a cycloaddition between the nitrene, formed from the alkylazide decomposition, and the carbon-carbon double bonds from rGO. 


\subsubsection{Analysis by FTIR and XPS spectroscopy}

To further assess the functionalization of $\mathrm{rGO}$, IR spectroscopy showed the appearance of new vibrational modes related to the agFc compound such as $\mathrm{C}-\mathrm{N}$ and $\mathrm{C}-\mathrm{O}$ bands at $\sim 1050-1150 \mathrm{~cm}^{-1}$ and an hyperchromic effect on the band located at ca. $550 \mathrm{~cm}^{-1}$ related to the Ring-TittTilt of torsional vibration of ferrocene [30] (Fig. $2 \in 2 d$ ). Moreover, the disappearance of the azide stretching band at $\sim 2100 \mathrm{~cm}^{-1}$ is in favor of the agFc and rGO coupling reaction [31]. Stronger proofs come from the XPS, in fact the wide scan survey of binding energy shows the presence of nitrogen (N1s at $\sim 401 \mathrm{eV}$ ) and iron (Fe2p at 711 and $\sim 725 \mathrm{eV}$ ) that can be ascribed to the aziridine and the ferrocene moieties, respectively (Fig. S3). Small amounts of iron oxide (FeOx) are observed at $\sim 709$ and $\sim 723 \mathrm{eV}$ and are due the degradation of ferrocene under harsh conditions of the coupling reaction (Fig. 4a) [32].
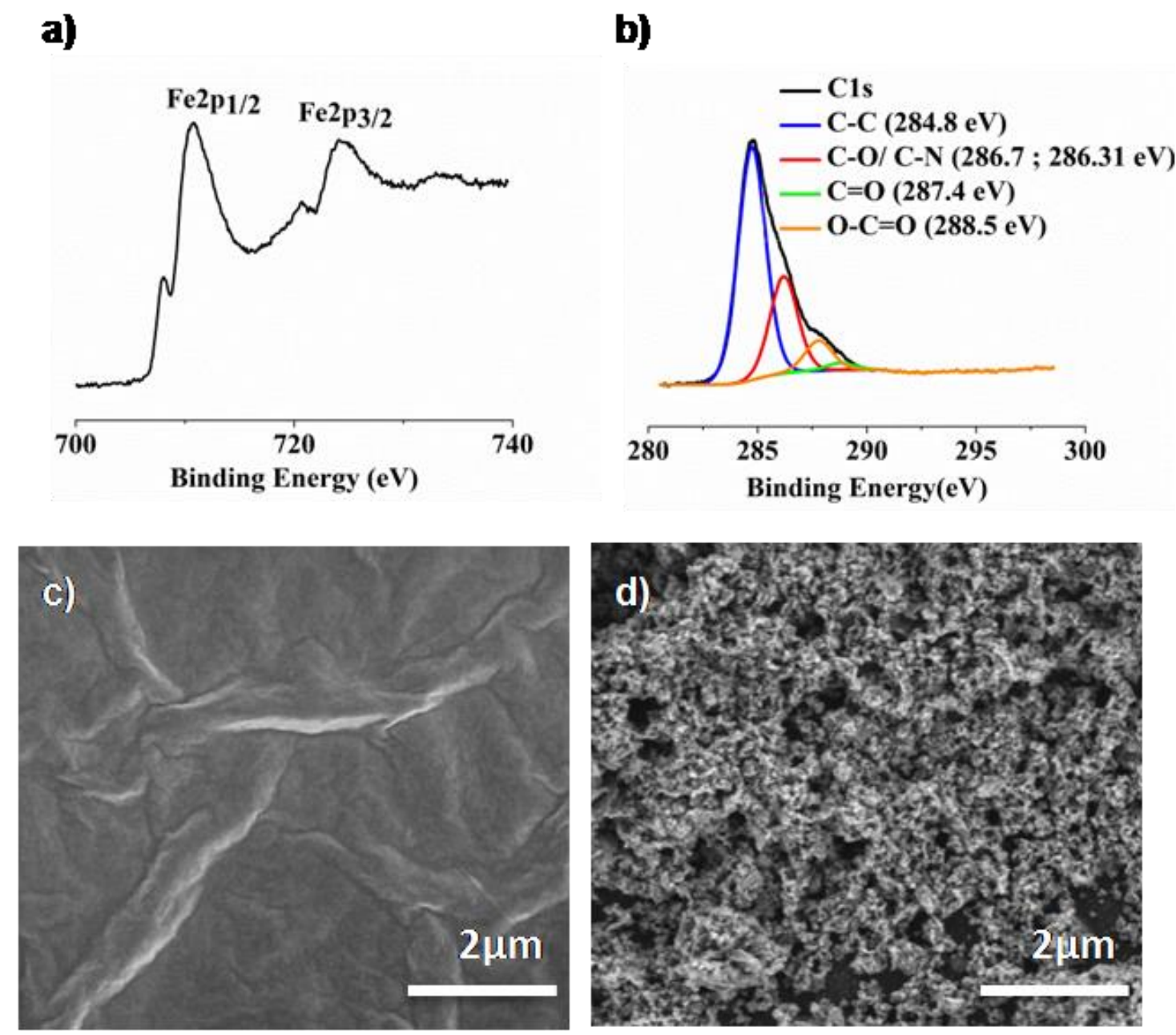

Fig. 4. The higher resolution XPS spectrum of Fe $2 p$ area of agFc-rGO (a); The deconvoluted XPS spectrum of the $C$ 1s peaks for agFc-rGO (b); SEM images of the $\mathrm{GO}$ (c) and agFc-rGO (d). 
Furthermore, more in-depth analysis of the carbon XPS signal shows the increase of contribution from $\mathrm{C}-\mathrm{O}$ and $\mathrm{C}-\mathrm{N}$ in agFc-rGO comparatively to that from $\mathrm{C}-\mathrm{O}$ in $\mathrm{rGO}$, the ratio increased to 0.62 from 0.51 , which allowed us to conclude the successful incorporation of agFc. (Fig. 4b). The quantification of the Fe-containing compounds based on Fe2p peak was not possible because of the difficulty to accurately determine the baseline [33]. Moreover, both $G O$ and rGO spectra revealed the presence of small amounts of nitrogen that could arise from impurities present in the commercial GO, and thus quantification of ferrocene moiety based on N1s peak is also not possible. EDX analysis revealed the presence of $\mathrm{Fe}, \mathrm{O}$ and $\mathrm{C}$ elements in the agFc-rGO nanosheets (Fig. S4). Despite that agFc-rGO is highly doped by iron compounds (17.7\%), the degree of agFc-rGO functionalization could not be estimated as $\mathrm{Fe}$ element originates from the coexistence of ferrocene and iron oxide.

\subsubsection{SEM and Contact angle measurement}

SEM images show that the ferrocene-functionalized $\mathrm{rGO}$ is completely crumpled and wrinkled compared to the starting material (Fig. 4c-d). Despite that the modification with ferrocene seems to diminish the grain size, the morphology of the unmodified rGO is still preserved after functionalization. Conversely, the agFc-rGO could not be easily characterized by SEM, which reveals the poor conductivity of functionalized rGO. This may be explained by the fact that [1,2]-cycloaddition leads to a band-gap opening in rGO and a transition from a semi-metallic to a semi-conducting state which can be attributed to the modification of the $\pi$-conjugated network that depends on the amount of the introduced aziridine groups [34]. This poor conductivity may also arise from the uncovered areas of the insulating PET substrate related to dropcasting methodology defects. Contact angle measurements showed that ferrocenefunctionalized graphene became more hydrophilic $\left(64^{\circ}\right)$ compared to $\mathrm{rGO}\left(98.3^{\circ}\right)$ (Fig. S1). This result is related to the increase of the oxidation level in agFc-rGO, already evidenced by XPS analysis, and is in agreement with results obtained with covalently functionalized carbon nanotubes [35].

\subsection{Preparation of the agFc-rGO electrode}

To prepare ferrocene-modified graphene electrode, the optimized amount (Fig. S5) of an aqueous agFc-rGO dispersion was drop-casted onto the PET substrate directly 
linked to a graphite connector and allowed to dry at RT (Fig. 5a). The drop dried into a ring-like shape, where most of the dispersed solid matter deposits along the perimeter showing the so-called "coffee-ring" effect. To circumvent this problem, agFc-rGO was dispersed into a CS solution. Then, $5 \mu \mathrm{L}$ of the resulting homogeneous solution was spread consistently onto PET and allowed to dry, resulting in the formation of a homogenous working electrode surface (Fig. 5b).
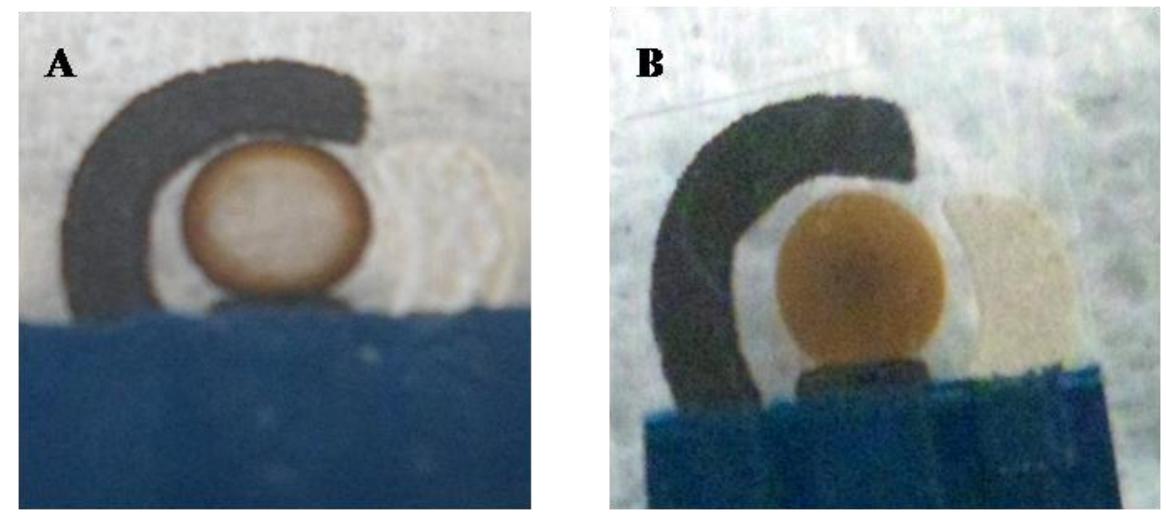

Fig. 5. Photographs of agFc-rGO electrode without $(A)$ and with $(B)$ addition of chitosan

To understand the influence of CS on the conductivity of agFc-rGO electrode, the electron-transfer kinetics of $\left[\mathrm{Fe}(\mathrm{CN})_{6}\right]^{3 / 4-}$ redox probe at agFc-rGO electrodes, prepared with and without CS, were studied using electrochemical impedance spectroscopy (Fig. S6). EIS plot for the agFc-rGO alone shows two semicircles that may correspond to the charge-transfer resistance levels at the edges and at the center (curve a). However, when dispersed in CS solution, the Nyquist plot shows only one semicircle and a linear portion at low frequency corresponding to the charge-transfer-limited process and the diffusion process, respectively (curve b). The experimental data were fitted using Randles equivalent circuit model where, $R s$ is electrolyte resistance, $R_{C T}$ is charge transfer resistance, $C_{d l}$ is double layer capacitance and $\mathrm{W}$ is Warburg diffusion impedance (inset of Fig. S6). The parameters extracted are gathered in Table $\mathrm{S} 1$. The charge transfer resistance (RCT) of the agFc-rGO electrode without CS was $2.98 \mathrm{k} \Omega$ and dropped by almost $30 \%$ when CS is added. This indicates a better electrical conductivity resulting from the better coverage and homogeneity of the working electrode surface.

To assess the novelty of this concept, two electrodes were prepared by drop-casting the same amount of agFc-rGO dispersion onto PET substrate and onto carbon 
working electrode of SPCE to form agFc-rGO electrode and agFc-rGO-modified SPCE, respectively. As shown in Fig. 6a, bare rGO and SPCE were nonelectroactive within the potential window. However, agFc-rGO-modified SPCE shows a strong ill-defined CV signal since the peak-to-peak separation exceeds $400 \mathrm{mV}$ and the $E_{1 / 2}$ potential was located at ca. $300 \mathrm{mV}$. This signal can be ascribed to the ferrocene moiety since the graphene-chitosan/nano- $\mathrm{Fe}_{3} \mathrm{O}_{4}$ composite [36] and graphene/ $\mathrm{Fe}_{3} \mathrm{O}_{4}$ composite [37] do not show redox signals in the selected potential window which, is another proof of the successful tethering of ferrocene into rGO. As for the agFc-rGO electrode, the overall current was lower but the ferrocene signal was well defined and the peak-to-peak separation dramatically decreased to $120 \mathrm{mV}$ along with the oxidation peak of the ferrocene from 500 to $300 \mathrm{mV}$ denoting a better electron-transfer. Moreover, the $\mathrm{E}^{1 / 2}$ shifted by $50 \mathrm{mV}$ to more cathodic potentials (ca. $0.25 \mathrm{~V}$ ). The low potential separation and the ipc-to-ipa ratio close to unity indicate a quasi-reversible electrode reaction. Stability studies of the agFc-rGO free-standing electrode exhibits good measurement stability by consecutive 20-cycle CV test, where the CV signal did not change and the ferrocene anodic peak current retained $99.8 \%$ of its initial current (Fig. S7). One can conclude that agFc-rGO electrodes are endowed with better electrochemical features since the signal is better defined. The use of agFc-rGO allows a better electron-transfer and avoids interferences from the carbon ink used in the screen-printing process. The lower current is probably due to the very low quantity of graphene used for the electrode preparation $(\sim 20 \mu \mathrm{g})$ compared to several mg used for screen-printing of carbon electrodes. Probably, the underlying conductive surface has influence on the ET rates. 


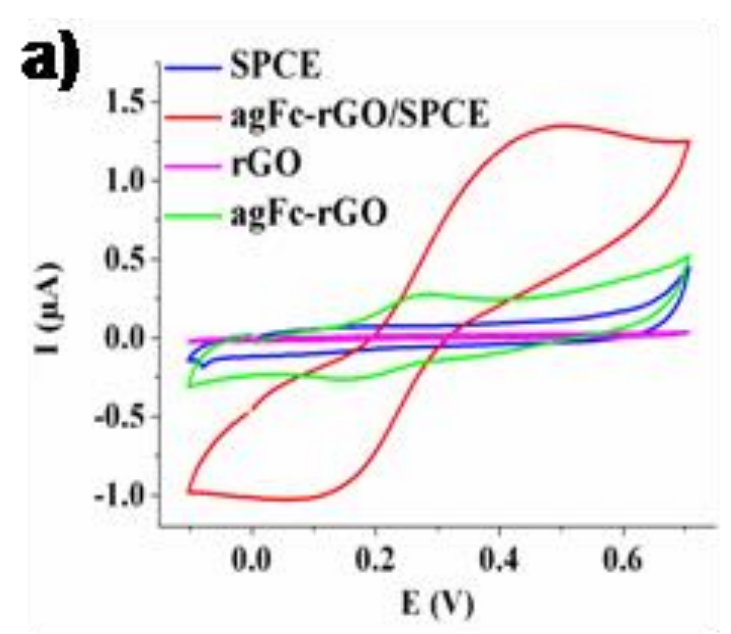

c)

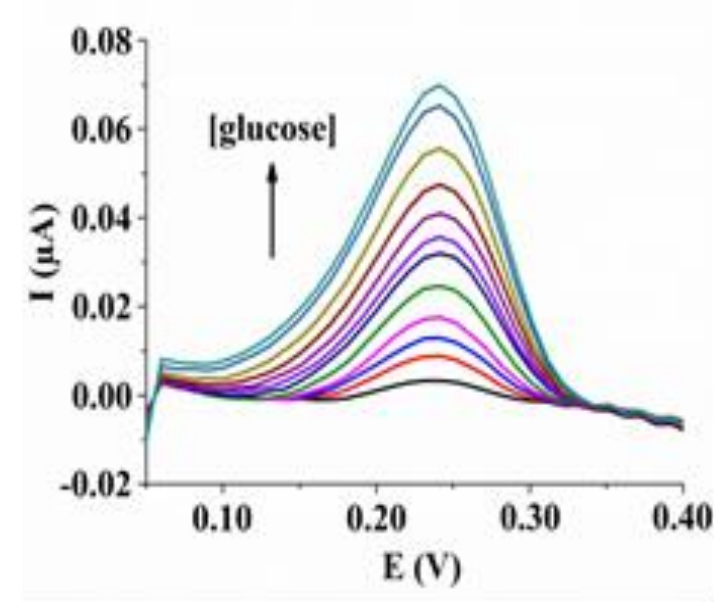

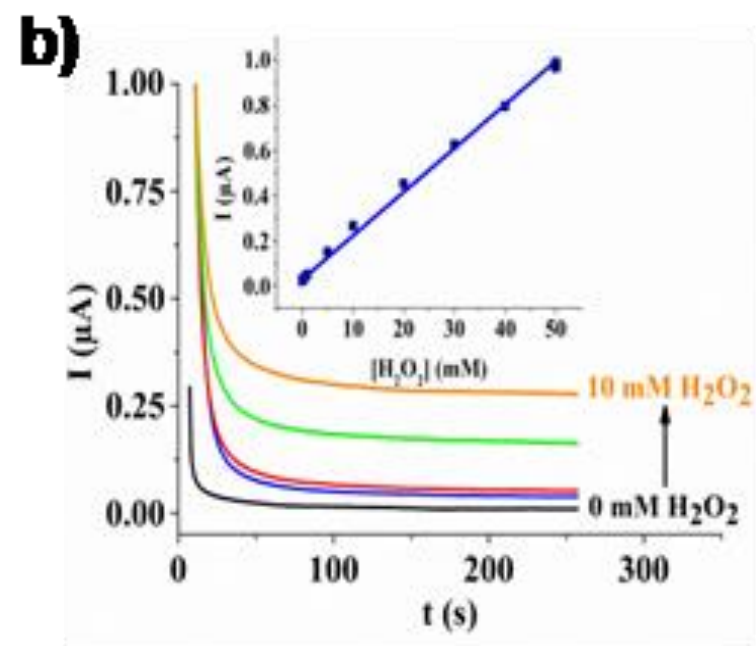

d)

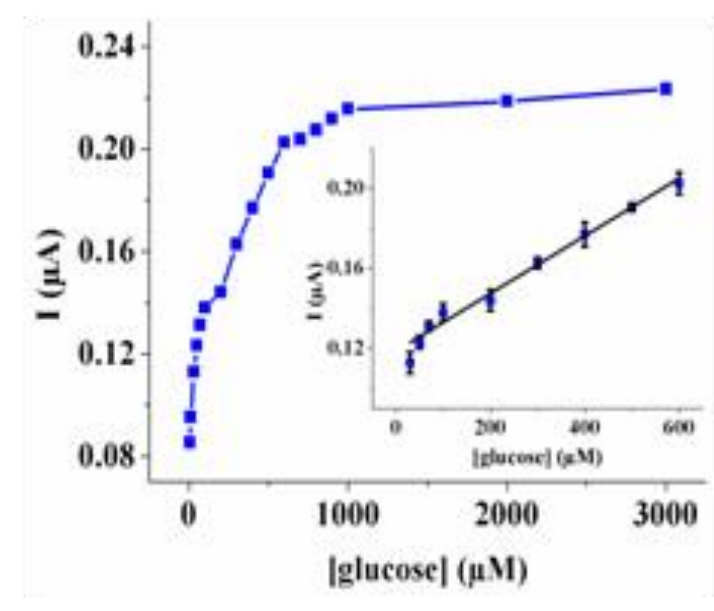

Fig. 6. a) Comparison of the CV signals obtained at SPCE (a), agFc-rGO modified SPCE (b), rGO electrode (c) and agFc-rGO electrode (d) in pH $7.4 \mathrm{PBS}$, at $100 \mathrm{mV} . \mathrm{s}^{-}$ 1 ; b) Amperometric responses of the agFc-rGO at $+600 \mathrm{mV}$ in $\mathrm{pH} 7.4$ PBS upon addition of $0,0.5 \mathrm{mM}, 1 \mathrm{mM}, 5 \mathrm{mM}$ and $10 \mathrm{mM} \mathrm{H}_{2} \mathrm{O}_{2}$, Inset: The linear range of $\mathrm{H}_{2} \mathrm{O}_{2}$ detection; c) DPV of the GOx/agFc-rGO electrode in pH 7.4 PBS with different concentrations of glucose from $5 \mu \mathrm{M}$ to $800 \mu \mathrm{M}$ at a scan rate of $100 \mathrm{mV} \cdot \mathrm{s}^{-1}$; d) The corresponding calibration curves of GOx/agFc-rGO electrode upon the successive addition of glucose, Inset: The linear range of glucose detection.

\subsection{Application of agFc-rGO electrode in $\mathrm{H}_{2} \mathrm{O}_{2}$ mediation}

The mediation of $\mathrm{H}_{2} \mathrm{O}_{2}$ oxidation and reduction was studied as a proof of concept in order to evaluate the electrocatalytic ability of agFc-rGO electrode and to benefit from the ferrocene for non-enzymatic $\mathrm{H}_{2} \mathrm{O}_{2}$ detection. $\mathrm{CV}$ shows that the addition of a large amount $\mathrm{H}_{2} \mathrm{O}_{2}$ induces an increase of both anodic and cathodic currents of the 
ferrocene while peak potentials remain unchanged (Fig. S8) suggesting an oxidation and reduction catalysis of $\mathrm{H}_{2} \mathrm{O}_{2}$. The amperometric responses of agFc-rGO electrode in $1 \mathrm{mM} \mathrm{H}_{2} \mathrm{O}_{2}$ at different potentials indicated that higher sensitivity is obtained at $+600 \mathrm{mV}$ (Fig. S9). The current recorded at this potential is three times higher than that observed at $+500 \mathrm{mV}$ denoting a potential-dependent electrocatalytic activity. Graphene-modified basal and edge plane pyrolytic graphite electrodes are able to lower the electrooxidation potential of $\mathrm{H}_{2} \mathrm{O}_{2}$ [38]. In fact, the oxidation current starts rising monotonously at ca. $+600 \mathrm{mV}$. In our case, at this potential, we can suggest the simultaneous oxidation of $\mathrm{H}_{2} \mathrm{O}_{2}$ via a ferrocene-mediated and a direct graphenecatalyzed processes explaining the increased sensitivity to $\mathrm{H}_{2} \mathrm{O}_{2}$. Upon successive addition of $\mathrm{H}_{2} \mathrm{O}_{2}$, a well-defined steady-state current plateau is reached rapidly with a wide linear range from 0.001 to $50 \mathrm{mM}$ (Fig. 6b). The detection limit was estimated to be $0.44 \mu \mathrm{M}(\mathrm{S} / \mathrm{N}=3)$ which is lower than that obtained with ferrocene-functionalized graphene modified GCE $(4.15 \mu \mathrm{M})$ [21] and with ferrocene-filled SWCNTs modified GCE (5 $\mu \mathrm{M})$ [39]. Moreover, the sensitivity was calculated to be $1267.32 \mu \mathrm{A} \cdot \mathrm{mM}^{-1} . \mathrm{cm}^{-}$ ${ }^{2}$ which is higher than that of Au NPs@POM-GNSs/GCE (58.87 $\left.\mu \mathrm{A} \mu \mathrm{A} \cdot \mathrm{mM}^{-1} \cdot \mathrm{cm}^{-2}\right)$ [40] and $\mathrm{RGO} / \mathrm{Fe}_{3} \mathrm{O}_{4} / \mathrm{Au}\left(688.00 \mu \mathrm{A} \mu \mathrm{A} \cdot \mathrm{mM}^{-1} \cdot \mathrm{cm}^{-2}\right)$ [41].

\subsection{Application of agFc-rGO electrode in glucose biosensing}

We also investigated the biofunctionalization of agFc-rGO electrode with glucose oxidase as a model enzyme. DPV showed an increase in ferrocene oxidation current upon successive additions of glucose (Fig. 6c). This behavior can be explained by the largely admitted Fc oxidation-mediated mechanism according to the following equations.

$\begin{array}{llll}\mathrm{GOx}(\mathrm{FAD})+\text { glucose } & \rightarrow & \mathrm{GOx}\left(\mathrm{FADH}_{2}\right)+\text { glucolactone } \\ \mathrm{GOx}(\mathrm{FADH} & 2)+2 \mathrm{Fc}^{+} & \rightarrow & \mathrm{GOx}(\mathrm{FAD})+2 \mathrm{Fc} \\ 2 \mathrm{Fc}(\text { electrode })+2 \mathrm{e}^{-} & \rightarrow & 2 \mathrm{Fc}^{+}(\text {electrode })\end{array}$

The electrocatalytic currents displayed a good linear behavior in the range from 30 to $600 \mu \mathrm{M}$ (Fig. 6d). The detection limit and the sensitivity were estimated to be $20 \mu \mathrm{M}$ $(\mathrm{S} / \mathrm{N}=3)$ and $9.396 \mu \mathrm{A} \cdot \mathrm{mM}^{-1} \cdot \mathrm{cm}^{-2}$, respectively. The biosensor exhibits lower detection limit than that of MWCNT/GO/GOx/GCE $(28 \mu \mathrm{M})$ [42] and RGO/Ag/GOx/GCE (160 $\mu \mathrm{M})$ [43]. Moreover, the sensitivity surpasses that of GOx- 
GQD/CCE $\left(0.085 \mu \mathrm{A} \cdot \mathrm{mM}^{-1} \cdot \mathrm{cm}^{-2}\right)$ [44] and RGO/Ag/GOx/GCE $\left(3.840 \mu \mathrm{A} \cdot \mathrm{mM}^{-1} \cdot \mathrm{cm}^{-2}\right)$ [43]. Since the physiological range of glucose concentrations found in human saliva is about 20-240 $\mu \mathrm{mM} \mathrm{[45],} \mathrm{the} \mathrm{biofunctionalized} \mathrm{agFc-rGO} \mathrm{electrode} \mathrm{can} \mathrm{be} \mathrm{used} \mathrm{as}$ a potential non-invasive probe for the glucose detection in saliva.

\section{Conclusions}

We have demonstrated that the functionalization of reduced graphene oxide by $\omega$ azidodiethyleneglycol $\alpha$-ferrocenyl-carboxylic acid ester, using nitrene chemistry, allowed preparing a ferrocene-modified graphene useful in graphene electrode preparation. The new agFc-rGO electrode structured platform is well designed to be used for the preparation of cost-effective and disposable sensors which have been applied for enzyme-free detection of $\mathrm{H}_{2} \mathrm{O}_{2}$ and enzymatic detection of glucose as model analytes. This work represents a preliminary investigation of functionalizedgraphene electrode preparation. In fact, and despite the limits presented by dropcasting methodology such as uncovered area, the results shown here could have wide implications since they demonstrate that it is possible to use a simple and general approach for functionalized-graphene electrode preparation.

\section{Acknowledgements}

ICN2 acknowledges support from the Severo Ochoa Program (MINECO, Grant SEV2013-0295) and MICINN for the Project MAT2011-25870 and Generalitat de Cataluña for SGR support. The Ministry of Higher Education and Scientific Research of Tunisia (MHESR), the UTM University and the LCAE laboratory are also acknowledged for research and travel funds granted for AR (LR99ES15).

\section{References}

[1] X. Zhu, Y. Liang, X. Zuo, R. Hu, X. Xiao, J. Nan, Novel water-soluble multinanopore graphene modified glassy carbon electrode for simultaneous determination of dopamine and uric acid in the presence of ascorbic acid, Electrochim. Acta 143 (2014) 366-373.

[2] S. Jiao, J. Jin, L. Wang, Tannic acid functionalized N-doped graphene modified glassy carbon electrode for the determination of bisphenol A in food package, Talanta 122 (2014) 140-144. 
[3] L. Baptista-Pires, B. Pérez-López, C. C. Mayorga-Martinez, E. Morales-Narváez, N. Domingo, M.J. Esplandiu, F. Alzina, C.M. Sotomayor Torres, A. Merkoçi, Electrocatalytic tuning of biosensing response through electrostatic or hydrophobic enzyme-graphene oxide interactions, Biosens. Bioelectron. 61 (2014) 655-662.

[4] A. R. Fakhari, A. Sahragard, H. Ahmar, Development of an electrochemical sensor based on reduced graphene oxide modified screen-printed carbon electrode for the determination of buprenorphine, Electroanalysis 26 (2014) 2474-2483.

[5] M. F. El-Kady, V. Strong, S. Dubin, R. B. Kaner, Laser scribing of highperformance and flexible graphene-based electrochemical capacitors, Science 335 (2012) 1326-1330.

[6] X. Shen, Y. Liu, Y. Pang, W. Yao, Conjugation of graphene on Au surface by $\pi-\pi$ interaction and click chemistry, Electrochem. Commun. 30 (2013) 13-16.

[7] K. R. Ratinac, W. Yang, J. J. Gooding, P. Thordarson, F. Braet, Graphene and related materials in electrochemical sensing, Electroanalysis 23 (2011) 803-826.

[8] I. N. Kholmanov, M. D. Stoller, J. Edgeworth, W. H. Lee, H. Li, J. Lee, C. Barnhart, J. R. Potts, R. Piner, D. Akinwande, J. E. Barrick, R. S. Ruoff, Nanostructured hybrid transparent conductive films with antibacterial properties, ACS Nano 6 (2012) 51575163.

[9] H. Feng, R. Cheng, X. Zhao, X. Duan, J. Li, A low-temperature method to produce highly reduced graphene oxide, Nat. Commun. 4 (2013) 1539.

[10] H. Zhou, W. Yao, G. Li, J. Wang, Y. Lu, Graphene/poly(3,4ethylenedioxythiophene) hydrogel with excellent mechanical performance and high conductivity, Carbon 59 (2013) 495-502.

[11] M. Chen, C. Zhang, X. Li, L. Zhang, Y. Ma, L. Zhang, X. Xu, F. Xia, W. Wang, J. Gao, A one-step method for reduction and self-assembling of graphene oxide into reduced graphene oxide aerogels, J. Mater. Chem. A 1 (2013) 2869-2877.

[12] L. Wang, X. Zhang, H. L.W. Chan, F. Yan, F. Ding, Formation and healing of vacancies in graphene chemical vapor deposition (CVD) growth, J. Am. Chem. Soc. 135 (2013) 4476-4482.

[13] E.P. Randviir, D.A.C. Brownson, J.P. Metters, R.O. Kadara, C.E. Banks, The fabrication, characterisation and electrochemical investigation of screen-printed graphene electrodes, Phys. Chem. Chem. Phys. 16 (2014) 4598-4611. 
[14] L. Huang, Y. Huang, J. Liang, X. Wan, Y. Chen, Graphene-based conducting inks for direct inkjet printing of flexible conductive patterns and their applications in electric circuits and chemical sensors, Nano Res. 4 (2011) 675-684.

[15] Y. Gao, W. Shi, W. Wang, Y. Leng, Y. Zhao, Inkjet printing patterns of highly conductive pristine graphene on flexible substrates, Ind. Eng. Chem. Res. 53 (2014) 16777-16784.

[16] K. A. Mkhoyan, A.W. Contryman, J. Silcox, D.A. Stewart, G. Eda, C. Mattevi, S. Miller, M. Chhowalla, Atomic and electronic structure of graphene-oxide, Nano Lett. 9 (2009) 1058-1063.

[17] H.J. Choi, S.M. Jung, J.M. Seo, D. W. Chang, L. Daic, J.B. Baek, Graphene for energy conversion and storage in fuel cells and supercapacitors, Nano Energy 1 (2012) 534-551.

[18] T. Gan, S. Hu, Electrochemical sensors based on graphene materials, Microchim. Acta 175 (1) (2011) 1-19.

[19] J. Ping, J. Wu, Y. Wang, Y. Ying, Simultaneous determination of ascorbic acid, dopamine and uric acid using high-performance screen-printed graphene electrode, Biosens. Bioelectron. 34(1) (2012) 70-76.

[20] S. Martic, M. Labib, P. O. Shipman, H. B. Kraatz, Ferrocene-peptido conjugates: From synthesis to sensory applications, Dalton Trans. 40(28) (2011) 7264-7290.

[21] L. Fan, Q. Zhang, K. Wang, F. Li, L. Niu, Ferrocene functionalized graphene: preparation, characterization and efficient electron transfer toward sensors of $\mathrm{H}_{2} \mathrm{O}_{2}$, J. Mater. Chem. 22 (2012) 6165-6170.

[22] R. S. Dey, R. Raj, Redox-functionalized graphene oxide architecture for the development of amperometric biosensing platform, ACS Appl. Mater. Interfaces 5 (2013) 4791-4798.

[23] K. Deng, J. Zhou, X. Li, Noncovalent nanohybrid of ferrocene with chemically reduced graphene oxide and its application to dual biosensor for hydrogen peroxide and choline, Electrochim. Acta 95 (2013) 18-23.

[24] G. Kalita, S. Sharma, K. Wakita, M. Umeno, Y. Hayashi, M. Tanemura, A photoinduced charge transfer composite of graphene oxide and ferrocene, Phys. Chem. Chem. Phys. 15 (2013) 1271-1274. 
[25] M. B. Gholivand, M. Khodadadian, Simultaneous voltammetric determination of captopril and hydrochlorothiazide on a graphene/ferrocene composite carbon paste electrode, Electroanalysis 25 (2013) 1263-1270.

[26] S. Tajik, M. A. Taher, H. Beitollahi, Application of a new ferrocene-derivative modified-graphene paste electrode for simultaneous determination of isoproterenol, acetaminophen and theophylline, Sens. Actuat. B 197 (2014) 228-236.

[27] S. Stankovich, D. A. Dikin, R. D. Piner, K. A. Kohlhaas, A. Kleinhammes, Y. Jia, Y. Wu, S. T. Nguyen, R. S. Ruof, Synthesis of graphene-based nanosheets via chemical reduction of exfoliated graphite oxide, Carbon 45 (2007) 1558-1565.

[28] H. J. Shin, K. K. Kim, A. Benayad, S. M. Yoon, H. K. Park, I. S. Jung, M. H. Jin, H.K. Jeong, J. M. Kim, J.Y. Choi, Y. H. Lee, Efficient reduction of graphite oxide by sodium borohydride and its effect on electrical conductance, Adv. Funct. Mater. 19 (2009) 1987-1992.

[29] A. Mars, C. Parolo, N. Raouafi, K. Boujlel, A. Merkoçi, Gold nanoparticles decorated with a ferrocene derivative as a potential shift-based transducing system of interest for sensitive immunosensing, J. Mater. Chem. B 1 (2013) 2951-2955.

[30] N. Mohammadi, A. Ganesan, C.T. Chantler, F. Wang, Differentiation of ferrocene $D_{5 d}$ and $D_{5 h}$ conformers using IR spectroscopy, J. Organometall. Chem. 713 (2012) 51-59.

[31] X. Xu, W. Lv, J. Huang, J. Li, R. Tang, J. Yan, Q. Yang, J. Qin, Z. Li, Functionalization of graphene by tetraphenylethylene using nitrene chemistry, RSC Adv. 2 (2012) 7042-7047.

[32] M. Pinault, M. Mayne-L'Hermite, C. Reynaud, V. Pichot, P. Launois, D. Ballutaud, Growth of multiwalled carbon nanotubes during the initial stages of aerosol-assisted CCVD, Carbon 43 (2005) 2968-2976.

[33] D. D. Hawn, B. M. DeKoven, Deconvalution as a correction for photoelectron inelastic energy losses in the core level XPS spectra of iron oxides, Surf. Interface Anal. 10 (1987) 63-74.

[34] K. Suggs, D. Reuven, X. Q. Wang, Electronic properties of cycloadditionfunctionalized graphene, J. Phys. Chem. C 115 (2011) 3313-3317.

[35] P. C. Ma, S. Y. Mo, B. Z. Tang, J. K. Kim, Dispersion, interfacial interaction and re-agglomeration of functionalized carbon nanotubes in epoxy composites, Carbon 48 (2010) 1824-1834. 
[36] H. Yin, Y. Zhou, Q. Ma, S. Ai, Q. Chen, L. Zhu, Electrocatalytic oxidation behavior of guanosine at graphene, chitosan and $\mathrm{Fe} 3 \mathrm{O} 4$ nanoparticles modified glassy carbon electrode and its determination, Talanta 82 (2010) 1193-1199.

[37] W. Qian, Z. Chen, S. Cottingham, W. A. Merrill, N. A. Swartz, A. M. Goforth, T. L. Clare, J. Jiao, Surfactant-free hybridization of transition metal oxide nanoparticles with conductive graphene for high-performance supercapacitor, Green Chem. 14 (2012) 371-377.

[38] W. J. Lin, C. S. Liao, J. H. Jhang, Y. C. Tsai, Graphene modified basal and edge plane pyrolytic graphite electrodes for electrocatalytic oxidation of hydrogen peroxide and $\beta$-nicotinamide adenine dinucleotide, Electrochem. Commun. 11 (2009) 21532156.

[39] N. Sun, L. Guan, Z. Shi, N. Li, Z. Gu, Z. Zhu, M. Li, Y. Shao, Ferrocene peapod modified electrodes: preparation, characterization, and mediation of $\mathrm{H}_{2} \mathrm{O}_{2}$, Anal. Chem. 78 (2006) 6050-6057.

[40] R. Liu, S. Li, X. Yu, G. Zhang, S. Zhang, J. Yao, B. Keita, L. Nadjo, L. Zhi, Facile Synthesis of Au-Nanoparticle/Polyoxometalate/ Graphene Tricomponent Nanohybrids: An Enzyme-Free Electrochemical Biosensor for Hydrogen Peroxide, Small 8(9) (2012) 1398-1406.

[41] Y. Ye, T. Kong, X. Yu, Y. Wu, K. Zhang, X. Wang, Enhanced nonenzymatic hydrogen peroxide sensing with reduced graphene oxide/ferroferric oxide nanocomposites, Talanta 89 (2012) 417-421.

[42] S. Palanisamy, S. Cheemalapati, S.M. Chen, Amperometric glucose biosensor based on glucose oxidase dispersed in multiwalled carbon nanotubes/graphene oxide hybrid biocomposite, Mater. Sci. Eng. C 34 (2014) 207-213.

[43] S. Palanisamy, C. Karuppiah, S.M. Chen, Direct electrochemistry and electrocatalysis of glucose oxidase immobilized on reduced graphene oxide and silver nanoparticles nanocomposite modified electrode, Colloids Surf. B: Biointerfaces 114 (2014) 164-169.

[44] H. Razmi, R. Mohammad-Rezaei, Graphene quantum dots as a new substrate for immobilization and direct electrochemistry of glucose oxidase: Application to sensitive glucose determination, Biosens. Bioelectron. 41 (2013) 498-504. 
[45] V. S. Siu, J. Feng, P. W. Flanigan, G. T. R. Palmore, D. Pacifici, A "plasmonic cuvette": dye chemistry coupled to plasmonic interferometry for glucose sensing, Nanophotonics 3 (2014) 125-140. 